\title{
Antimitochondrial antibody negative primary biliary cirrhosis: a distinct syndrome of autoimmune cholangitis
}

\author{
P Michieletti, I R Wanless, A Katz, P J Scheuer, S J Yeaman, M F Bassendine, J M Palmer, \\ E J Heathcote
}

\begin{abstract}
This study reports on a group of 20 patients with an initial diagnosis of primary biliary cirrhosis (PBC) whose serum tested negative for antimitochondrial antibodies by immunofluorescence. All had a clinical history compatible with primary biliary cirrhosis, and results of biochemical, histological, and radiological investigations were consistent with this diagnosis despite the absence of antimitochondrial antibodies by immunofluorescence. For comparison, these patients were matched for sex and serum bilirubin with 20 antimitochondrial antibody positive $(>1: 160)$ and histologically confirmed primary biliary cirrhosis patients who served as controls. Serum samples from both groups were retested blindly for antimitochondrial antibodies using immunoblotting and for antibodies to the major M2 mitochondrial autoantigens by enzyme linked immunosorbent assay (ELISA). Three antimitochondrial antibody immunofluorescence negative patients had antimitochondrial antibodies by immunoblotting and ELISA; the remaining 17 patients were confirmed negative by all methods. The antimitochondrial antibody immunofluorescence positive controls were verified by immunoblotting or ELISA, or both. All 17 patients negative for antimitochondrial antibodies had antinuclear antibodies, often in high titres, compared with 3/17 of the antimitochondrial antibody positive controls $(p=0.0001)$. Additionally, the antimitochondrial antibody negative group also had significantly higher smooth muscle antibody titres $(p=0.03)$ and lower serum $\operatorname{Ig} M(p=0.01)$ and aspartate aminotransferase $(p=0.03)$ activities than the antimitochondrial antibody positive controls. Analysis of clinical findings, histological tests, serum bilirubin, alkaline phosphatase, alamine aminotransferase, and IgG, disclosed no significant differences between the two groups. This paper describes a group of patients with the clinical and histological features of PBC but who do not fulfil the usual criteria necessary to make this diagnosis. Because they also have very high titres of antinuclear antibodies, smooth muscle antibodies, and comparatively low IgM and aspartate aminotransferase activities, we believe they are distinct from PBC and have a syndrome of autoimmune cholangitis.

(Gut 1994; 35: 260-265)
\end{abstract}

Primary biliary cirrhosis (PBC) is a chronic progressive liver disease of unknown cause. A positive diagnosis in patients who may or may not be symptomatic, usually requires the combination of an increase in serum alkaline phosphatase and immunoglobulin $M$ (IgM), together with serum antimitochondrial antibodies (AMA) and histological evidence of bile duct injury and portal inflammation.

Most patients with PBC (greater than $80 \%$ ) have AMA when serum samples are tested with the immunofluorescence technique (AMAIF).$^{1-4}$ In one series of 218 patients, nine were AMA-IF negative and seven of these were subsequently found to be AMA-IF positive when additional serum specimens were examined suggesting that less than $1 \%$ of $\mathrm{PBC}$ patients were negative for AMA-IF in this large study. ${ }^{+}$The identification and purification of the major mitochondrial autoantigens has permitted immunoassays to be developed that are more sensitive than immunofluorescence techniques for the detection of AMA. ${ }^{5-10}$

In this study we report the clinical, serological, and histological features of patients initially thought to have PBC by all the accepted criteria except they proved to be AMA-IF negative. Our findings show that patients with AMA-negative $\mathrm{PBC}$ may have a syndrome immunologically distinct from AMA-positive PBC.

\section{Methods}

\section{PATIENTS}

The subjects in this study are 20 Canadian patients (primarily from southern Ontario) with an initial clinical diagnosis of PBC who were referred for possible inclusion into a therapeutic trial of ursodeoxycholic acid, but were ineligible because their serum samples were AMA-IF negative and thus they were not treated. We selected a comparison group of 20 AMA-IF positive (titre $>1: 160$ ) PBC patients who were referred to the same trial and used their serum (before entering the trial) for this study. These controls were selected because they matched the study subjects for sex and serum bilirubin at the time of referral. Testing for serum antimitochondrial antibodies by the methods described below, was then performed on the index patients and controls. The group of patients who were confirmed AMA negative by all testing methods (referred to as AMA-all negative) were then compared with the matched AMA positive (AMA-all positive) controls for the presence of other non-organ specific antibodies, clinical features, serum liver biochemistry, and percu- 
taneous liver biopsy specimens, which were obtained from patients and controls near the time of referral to the trial.

\section{SEROLOGICAL TESTS}

Serum was tested by indirect immunofluorescence for the presence of antimitochondrial (AMA-IF), antinuclear, and smooth muscle antibodies. Frozen sections of rat kidney and stomach as well as a Hep-2 cell line (Kallestad Diagnostics, Chaska MN) were used as substrate. Serum was screened at a dilution of $1 / 20$ and 1/40 and further dilutions were tested when indicated. The conjugate used was fluorescein conjugated rabbit anti-human IgA, IgG, IgM, kappa and lambda (Dakopotts, Glostrup Denmark), which had a protein concentration of $4.2 \mathrm{~g} / \mathrm{l}$ and a fluorescein/protein molar ratio of $2 \cdot 3$. The dilution of the conjugate used was $1 / 160$ in phosphate buffered saline as determined by chessboard titration. " Each section and tissue culture was examined by two pathologists using a Leitz Orthoplan fluorescent microscope with a Ploemopak 2 vertical illuminator equipped with water immersion objectives.

Serum samples were tested for AMA by immunoblotting. For this assay, pyruvate dehydrogenase complex, 2-oxo-glutarate dehydrogenase complex was purified from bovine heart, ${ }^{12}$ and branched chain 2-oxo acid dehydrogenase complex was purified from rat liver (gift of Dr Y Shimomura, University of Nagoya, Japan). ${ }^{13}$ Western immunoblotting against these three complexes was performed as previously described ${ }^{14}$ at a serum dilution of $1: 100$ and $1: 1000$ and using $2 \mu \mathrm{g}$ of each complex per lane. Reactivity was visualised using peroxidase conjugated IgG and IgM specific secondary antibodies (Sigma Chemical Co, St Louis, Mo) with 4-chloro-1-naphthol as substrate. ${ }^{15}$

Samples were also tested for AMA by an ELISA using purified mitochondrial antigens as previously described. ${ }^{8}$ The E2 component together with protein $\mathrm{X}$ of the pyruvate dehydrogenase complex was obtained by resolution of the complex using gel filtration chromatography in the presence of $1 \mathrm{M} \mathrm{NaCl}$.

The liver specimens were fixed in buffered formalin, embedded in paraffin wax, and sections stained with haematoxylin and eosin, Masson's trichrome, orcein, PAS after diastase, and by the Prussian blue method. Slides were reviewed independently by two pathologists (IRW and PJS) who had no knowledge of the AMA results; for each biopsy specimen a histological diagnosis was assigned as definite $\mathrm{PBC}$, probable $\mathrm{PBC}$, non-specific but possible $\mathrm{PBC}$, or

TABLE I Summary of antimitochondrial antibody (AMA) investigations

\begin{tabular}{lll}
\hline Method & $\begin{array}{l}\text { AMA-IF negative patients } \\
\text { testing positive }\left(n=20^{\star}\right)\end{array}$ & $\begin{array}{l}\text { AMA-IF positive patients } \\
\text { testing positive }\left(n=20^{\star}\right)\end{array}$ \\
\hline Immunofluorescence & 0 & 20 \\
ELISA for E2/X & 3 & 20 \\
Immunoblotting for PDC & 2 & 20 \\
Immunoblotting for OGDC & 0 & 2 \\
Immunoblotting for BCOADC & 1 & 8 \\
\hline
\end{tabular}

$\mathrm{PDC}=$ pyruvate dehydrogenase complex $\mathrm{OGDC}=2$-oxo-glutarate dehydrogenase complex; $\mathrm{BCOADC}=$ branch chain 2-oxo acid dehydrogenase complex; ${ }^{\star}$ for $\mathrm{BCOADC}, \mathrm{n}=14$. other diagnosis. One pathologist (IRW), graded the histological parameters of fibrosis, inflammation, duct paucity, and duct necrosis using a scale of 0 to 3 (none, mild, moderate, and severe). Granulomas were noted as present or absent.

\section{STATISTICAL ANALYSIS}

The differences between the AMA negative patients and their AMA positive controls were compared using the Wilcoxon rank sign test for various clinical and histological features and the serum measurements of alkaline phosphatase, aminotransferases, antinuclear titres, smooth muscle antibodies titres, $\operatorname{IgM}$, and IgG.

\section{Results}

Seventeen of 20 (85\%) AMA-IF negative patients were confirmed to be also negative for AMA by ELISA for E2/X and immunoblotting (will be referred to as AMA-all negative) (Table I). Three of 20 AMA-IF negative patients (15\%) were found to have antibodies to pyruvate dehydrogenase complex E2/protein $X$ by ELISA; testing with immunoblotting verified IgM and IgG antibodies to pyruvate dehydrogenase complex in two of these patients and branch chain 2-oxo acid dehydrogenase complex antibodies in the other. All 20 AMA-IF positive control patients were confirmed AMA positive for pyruvate dehydrogenase complex E2/X by ELISA (AMA-all positive).

Recognising that the detection of AMA-IF increases as patients are retested over time,${ }^{4}$ we performed at least three immunofluorescence tests on separate samples in the 20 AMA-IF negative patients; 19 continued to be negative over several years. The only exception was one patient initially testing AMA-IF negative who became AMA-IF positive after two years. On retesting the initial serum of this patient, it was positive by ELISA and immunoblotting. Two other AMA-IF negative but ELISA positive patients who have been followed up for five and three years respectively continue to test AMA-IF negative.

Further investigations showed that all of the 17 AMA-all negative patients were antinuclear antibody positive; eight with a titre of $1: 160$ $1: 1280$, eight at $1: 40-1: 80$, and one at $1: 20$ (Table II). Three of 17 AMA-all negative patients were positive for antinuclear antibodies only on rat kidney substrate while the remaining 14 were positive in both rat kidney and Hep 2 cells. This was considerably different from the antinuclear antibody titres in the 17 AMA-all positive patients $(p=0.0001)$, all but three of whom were antinuclear antibody negative (titres $<1: 20)$. No consistent immunofluorescence staining pattern was seen in either group. Smooth muscle antibody was also more prevalent in the AMA negative group ( $30 v 5 \%, \mathrm{p}=0.03)$.

Eighty two per cent (14/17) of the AMA-all negative patients were female. All 17 had an abnormal alkaline phosphatase, four an abnormal bilirubin, and 10 an increased $\operatorname{IgM}$ value. Cholangiography was attempted in all the AMAall negative patients and was successful in 14, all 
TABLE II Summary of antinuclear antibody (ANA) titres, immunofluorescence patterns, and smooth muscle antibody (SMA) titres

\begin{tabular}{lll}
\hline & $\begin{array}{l}\text { AMA-all negative } \\
\text { patients }(n=17)\end{array}$ & $\begin{array}{l}\text { AMA-all positive } \\
\text { patients }(n=17)\end{array}$ \\
\hline ANA titres: & & \\
1:160-1:1280 & 8 & 2 \\
1:40-1:80 & 8 & 0 \\
1:20 & 1 & 1 \\
$\quad$ Total & $17^{\star}$ & 3 \\
Patterns: & 6 & 0 \\
$\quad$ Homogeneous & 2 & 2 \\
Speckled & 3 & 1 \\
Anti-centromere & 2 & 0 \\
Multiple nuclear dot & 1 & 0 \\
Rim & 3 & 0 \\
Indeterminant & & 1 \\
SMA titres: & 4 & 0 \\
1:20-1:40 & 3 & 1 \\
1:80-1:160 & $7 \star$ & \\
Total & & \\
\hline
\end{tabular}

${ }^{\star} \mathrm{p}=0.0001$ for ANA, $\mathrm{p}=0.03$ for SMA.

TABLE III Summary of clinical parameters

\begin{tabular}{lll}
\hline $\begin{array}{l}\text { Parameters at time } \\
\text { of referral }\end{array}$ & $\begin{array}{l}\text { AMA-IF negative } \\
\text { patients }(n=17)\end{array}$ & $\begin{array}{l}\text { AMA-IF positive } \\
\text { patients }(n=17)\end{array}$ \\
\hline $\begin{array}{ll}\text { Female:male ratio } \\
\text { Age at diagnosis (mean, } \\
\text { range) }\end{array}$ & $14: 3$ & $14: 3$ \\
Jaundice & $51 \cdot 1(35-63)$ & $55 \cdot 0(31-79)$ \\
Fatigue & 3 & 3 \\
Pruritus & 8 & 7 \\
Xanthelasma & 9 & 11 \\
Hypothyroidism & 3 & 1 \\
Polyarthralgias & 3 & 3 \\
Sicca syndrome & 5 & 3 \\
CREST syndrome & 3 & 7 \\
Raynaud's phenomenon & 0 & 1 \\
Insulin dependent diabetes & 2 & 4 \\
& 2 & 1 \\
\hline *p 0.05 for all parameters & &
\end{tabular}

of whom had normal extrahepatic bile ducts. No patient had a history of diarrhoea, ulcerative colitis or spondyloarthropathy. No patient had been treated with drugs known to cause bile duct paucity or with corticosteriods. After the diagnosis of AMA-IF negative PBC, no patient was treated with any drugs except cholestyramine for pruritus.

Comparison of the clinical findings showed no significant difference between the AMA-all negative and AMA-all positive patients with respect to signs, symptoms, and associated autoimmune phenomena at the time of diagnosis (Table III). Similarly, serum IgG, alkaline phosphatase, and alanine aminotransferase activities at the time of referral were not significantly different (Table IV). The serum IgM and aspartate aminotransferase, however, were significantly lower in the AMA-IF negative group $(p=0.01$ and $p=0.03$ respectively).

The mean follow up time in the AMA-all negative and positive patients was $4.8 v 4 \cdot 4$ years
TABLE IV Summary of laboratory parameters

\begin{tabular}{lcc}
\hline $\begin{array}{l}\text { Parameters at time } \\
\text { of referral (normal range })\end{array}$ & $\begin{array}{c}\text { AMA-IF negative patients } \\
(n=17)(\text { mean }(\text { median, range }))\end{array}$ & $\begin{array}{l}\text { AMA-IF positive patients } \\
(n=17)(\text { mean }(\text { median, range }))\end{array}$ \\
\hline Total bilirubin $(<20 \mu \mathrm{mol} / \mathrm{l})$ & $43(12,7-309)$ & $38(13,7-239)$ \\
ALP $(<100 \mathrm{IU} / 1)$ & $500(279,109-1225)$ & $374(350,106-880)$ \\
AST $(<35 \mathrm{IU} / /)$ & $79(63,22-176)^{\star}$ & $112(90,24-234)$ \\
ALT $(<40 \mathrm{IU} / \mathrm{I})$ & $94(61,20-216)$ & $124(101,18-297)$ \\
IgG $(5 \cdot 5-15 \cdot 6 \mathrm{~g} / 1)$ & $17 \cdot 75(15 \cdot 9,9 \cdot 3-29 \cdot 5)$ & $16 \cdot 8(14.6,9-36 \cdot 1)$ \\
IgM $(0 \cdot 4-3 \cdot 2 \mathrm{~g} / \mathrm{l})$ & $3 \cdot 6(3 \cdot 2,0 \cdot 5-6 \cdot 6)^{\star}$ & $6 \cdot 4(4 \cdot 9,1 \cdot 9-17 \cdot 4)$ \\
\hline
\end{tabular}

${ }^{\star} p=0.01$ for IgM, $p=0.03$ for AST, other parameters not significantly different. ALP=alkaline phosphatase; AST =aspartate aminotransferase; ALT=alanine aminotransferase.

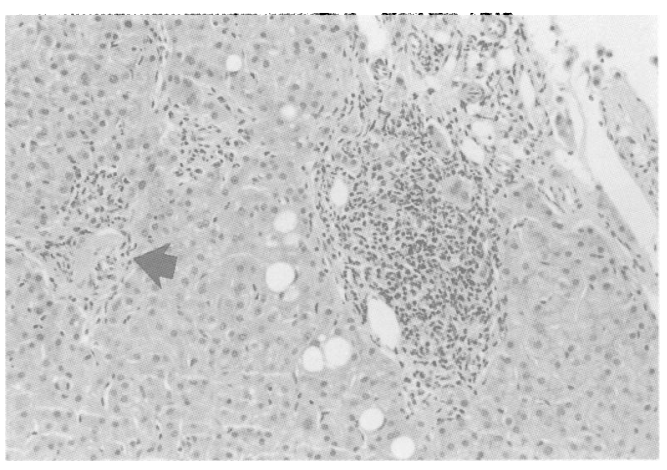

Figure 1: Two portal tracts are shown. The left (arrow) has a minimal inflammatory infiltrate and absence of ducts. The right portal tract has a heavy lymphoid infiltrate, intact limiting plate, and duct obscured by the infiltrate.

(Haematoxylin and eosin, original magnification $\times 220$.)

respectively. One AMA-all negative patient received a liver transplant and another died of liver failure while two patients in the AMApositive group received liver transplants. Nine AMA-all negative patients and 11 AMA-all positive patients had symptoms of pruritus, jaundice or xanthelasma at the time of diagnosis. A further three AMA-all negative patients and one AMA-all positive patient who were asymptomatic at diagnosis subsequently developed pruritus or xanthelasma, or both over the follow up period up to five years after diagnosis. Ten AMA-all negative and nine AMA-all positive patients had at least one associated autoimmune phenomenon at the time of diagnosis. One more AMA-all negative and three AMA-all positive patients subsequently developed a new associated autoimmune disease. Hence there were no clinical features that distinguished the two groups.

All 17 biopsy specimens from the AMA-all negative patients showed a portal inflammatory infiltration that was mixed with a predominance of lymphocytes and smaller numbers of plasma cells, eosinophils, and neutrophils. Within a given biopsy specimen the infiltrate often varied and tracts with active duct injury usually had more severe infiltration with lymphoid and granulocytic cells evenly represented (Fig 1). Nodular lymphoid aggregates without germinal centres were seen in one biopsy specimen. Mild parenchymal lymphoid infiltration was present in seven specimens, often associated with focal

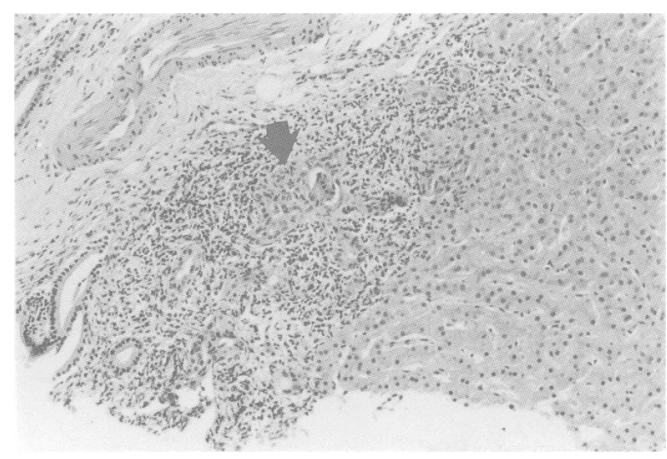

Figure 2: There is a focal predominantly lymphoid infiltrate confined to the region of a duct that is infiltrated and focally necrotic (arrow). Other ducts on the left are normal.

(Haematoxylin and eosin, original magnification $\times 175$.) 


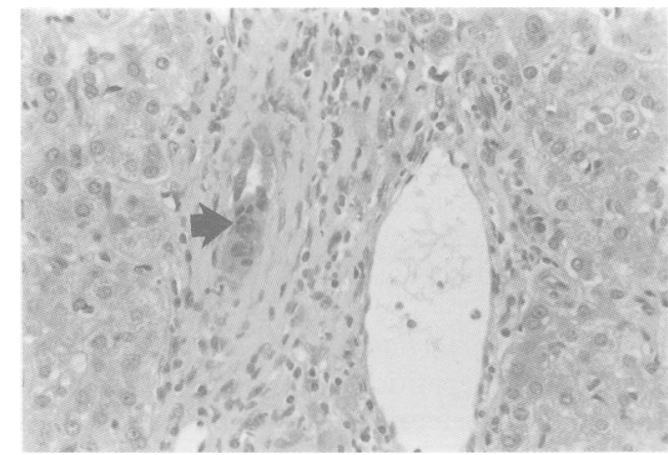

Figure 3: An injured duct has multilayering of epithelium (arrow) with minimal surrounding inflammatory infiltrate. (Haematoxylin and eosin, original magnification $\times 580$.)

drop out of hepatocytes without ballooning or acidophilic bodies. Nine specimens had ductual proliferation. Four of these had 'biliary piecemeal necrosis' with a mixed infiltrate and, in one specimen, periportal feathery degeneration was seen. No specimen had visible bile pigment and Mallory bodies were not seen. Duct necrosis was prominent in two specimens (Figs 2-4) and subtle in eight. Bile duct paucity was found in all specimens and more than half of portal tracts were without ducts in 12 specimens. Poorly demarcated collections of epithelioid macrophages were found adjacent to damaged ducts in two specimens and one specimen had a small parenchymal granuloma. Portal oedema was prominent in four, usually in the presence of duct injury and mild infiltrate of eosinophils and lymphocytes. Periductal oedema produced an onion skin appearance in one specimen. Cirrhosis was present in two specimens and lesser degrees of portal fibrous expansion were found in 12 .

Both pathologists agreed that the patients who were AMA-all negative, had histological findings that were qualitatively indistinguishable from those seen in AMA-positive PBC. Furthermore, quantitative differences were not seen except for a slightly greater degree of portal inflammation in the AMA-positive cases (Table V).

\section{Discussion}

We describe a group of 20 predominantly female patients, who have the clinical, biochemical, and histological appearance of PBC, but who did not have AMA by immunofluorescence despite repeated investigations during several years of

TABLE V Summary of histological parameters

\begin{tabular}{lcc}
\hline & $\begin{array}{c}A M A \text {-all negative patients } \\
(n=17)\end{array}$ & $\begin{array}{l}\text { AMA-all positive patients } \\
(n=17)\end{array}$ \\
\hline Histological diagnosis: & & \\
Definite or likely PBC & 7 & 10 \\
Non-specific, compatible with PBC & 10 & 7 \\
Other diagnosis & 0 & 0 \\
Specific histological parameters: & 2 & 0 \\
Duct necrosis (grade 2-3) & 9 & 8 \\
Duct paucity (grade 2-3) & 7 & 14 \\
Portal inflammation (grade 2-3) & 3 & 7 \\
Granulomata (present) & 8 & 7 \\
Fibrosis (grade 2-3) & & \\
\hline
\end{tabular}

${ }^{\star} \mathrm{p}=0.02$ for portal inflammation; all other comparisons not significant.

$\mathrm{PBC}=$ primary biliary cirrhosis.



Figure 4: This necrotic duct has a single recognisable cell (arrow) surrounded by an intense lymphoid infiltrate. (Haematoxylin and eosin, original magnification $\times 400$.)

follow up. Reports in published works suggest that $1-16 \%$ of $P B C$ patients do not have AMA by immunofluorescence. ${ }^{14}$ Our group of 20 AMAIF negative patients was initially identified from a cohort of 330 patients with PBC who were referred for a clinical trial. This apparent prevalence of $6 \%$, however, may be falsely low, because we initially canvassed for patients who were AMA positive.

Application of the more sensitive and specific assays of immunoblotting and ELISA for detection of AMA in the 20 AMA-IF negative patients, confirmed that $17(85 \%)$ of these patients are truly AMA negative.

The 17 AMA-all negative patients and their controls were comparable in all respects including clinical findings, prevalence of autoimmune diseases, serum bilirubin, alkaline phosphatase, alanine aminotransferase, and IgG values. Because these AMA-all negative patients, however, truly lack antimitochondrial antibodies and have a comparatively low IgM concentration, they do not fulfil the criteria generally used to make the diagnosis of classic PBC. Additionally, five significant differences were present in the AMA-all negative patients; they were more likely to have antinuclear antibodies (frequently in high titre), smooth muscle antibodies, a lower serum IgM concentration, lower serum aspartate aminotransferase concentration, and less portal inflammation in their liver biopsy specimens.

Patients with AMA-IF positive PBC have also been found to have antinuclear antibodies with a prevalence of 0 to $46 \% .{ }^{17-21}$ Our AMA-all positive controls had a prevalence of $15 \%$. This is appreciably different from the $100 \%$ prevalence found in our 17 AMA-all negative patients. A recent study in PBC examining antinuclear antibodies to a nuclear envelope protein, showed that the presence of this family of antibodies is not specific for a subset of patients with a particular pattern of clinical, biological or histological features. ${ }^{21}$

Specific antinuclear antibody subtypes have been described in $\mathrm{PBC}$, but none of these subtypes was overrepresented in our AMA-all negative cases. For example, antibodies with a nuclear rim pattern have been reported in about half of antinuclear positive $\mathrm{PBC}$ patients and in 3/4 AMA-IF negative PBC patients. ${ }^{22}$ We have not seen this pattern in AMA positive $\mathrm{PBC}$ patients and it was present in 1/17 AMA-all negative patients. Similarly, the multiple nuclear 
dot pattern seemed to be overrepresented in AMA-IF negative PBC in one study..$^{23} \mathrm{We}$ found this pattern in only two of $17 \mathrm{AMA}$-all negative patients.

The presence of antinuclear and antismooth muscle antibodies raises the question of autoimmune chronic active hepatitis in our AMA-all negative patients. We believe this is unlikely as the symptoms and clinical findings were typical of cholestasis not hepatitis, and their serum aminotransferase values were not in the hepatitic range. In fact, the aspartate aminotransferase was significantly lower in the AMA-all negative patients than in the PBC control group. Additionally, the AMA-all negative liver biopsy specimens did not have periportal inflammation and necrosis ('piecemeal necrosis'), but did have the histological features of duct injury and granulomas, which are not often seen in chronic active hepatitis.

The overlap syndrome of PBC and autoimmune chronic aggressive hepatitis was suggested by Kloppel et al. ${ }^{24}$ They described 27 AMA positive patients, 13 of whom had antibodies directed against the outer mitochondrial membrane. They frequently had positive antinuclear antibodies and had biopsy specimens that showed parenchymal injury and piecemeal necrosis. A similar AMA positive and antinuclear antibody positive patient has also been described who had features of both PBC and chronic active hepatitis on liver biopsy examination. ${ }^{25}$ Recently, there was a report of an AMA negative, antinuclear antibody and smooth muscle antibody positive woman with the clinical and pathological features of both autoimmune chronic aggressive hepatitis and PBC. ${ }^{26}$ Upon treatment with prednisone, her clinical course improved. The patients described in these publications were clearly very different from ours in that they were either AMA positive, or had high aminotransferases and liver biopsy specimens showing piecemeal necrosis and parenchymal injury, or all three.

Bile duct injury and loss has been reported in a variety of clinical settings in addition to PBC, including primary sclerosing cholangitis, graft $v$ host disease, graft rejection, and drug toxicity. An important paper by Ludwig et al has reported the existence of adult onset ductopoenia in the absence of these conditions. ${ }^{27}$ There are approximately 13 patients reported to date with adult onset idiopathic ductopoenia in the absence of AMA. ${ }^{27-30}$ Unlike our AMA-all negative population these patients were often men, did not have evidence of ulcerative colitis, did not have hepatic granulomas, and duct necrosis was usually not seen. No reference to the presence or absence of autoantibodies was made.

Brunner et al reported three AMA-IF negative women who had ductopoenia with antinuclear in high titre. ${ }^{31}$ Histological examination showed a granuloma in one patient and duct necrosis in another, confirming a PBC like syndrome. After immunosuppressive treatment with prednisone and azathioprine, the liver enzymes improved and the term 'immunocholangitis' was applied to these patients. Available data suggest that these three patients had a similar disease pattern to our AMA negative patients. Our patients, however, have been treated with only palliative measures to date.

There is evidence suggesting that AMA in the form of autoantibodies against the pyruvate dehydrogenase-E2 may be the T-cell epitope, which may initiate the $T$-cell response leading to biliary epithelial damage. Monoclonal mouse antibodies, which react to $\mathrm{PDH}-\mathrm{E} 2$ produce distinct intense activity when used to stain bile ducts in patients with $\mathrm{PBC}$ in contrast with bile duct specimens from patients with primary sclerosing cholangitis and autoimmune chronic active hepatitis. ${ }^{32}$ Cloned T-cell lines have been generated from patients with $\mathrm{PBC}$, but not from those with chronic aggressive hepatitis, which produce IL-2 specifically in response to $\mathrm{PDH}$ E2. In contrast, our results suggest that the clinical and histological pattern typical of $\mathrm{PBC}$ is quite independent of any evidence of serum antibodies for AMA. This may signify that AMA play no part in the pathogenesis of $\mathrm{PBC}$ or that the clinical and histological features we would normally associate with AMA positive $\mathrm{PBC}$ are a non-specific response to an immune liver injury.

In conclusion, there are now several syndromes with features of apparent autoimmune liver injury including primary biliary cirrhosis, primary sclerosing cholangitis, and autoimmune chronic active hepatitis. Our 17 AMA-all negative patients were initially thought to have PBC based on their symptoms and histological tests. They did not have, however, the immune markers associated with PBC (AMA and raised IgM), two of four criteria commonly used to make the diagnosis of PBC. Instead they have antinuclear antibodies often at very high titres, smooth muscle antibodies, and comparatively low serum IgM and aspartate aminotransferase activities. We believe that these AMA-all negative patients have a form of autoimmune cholangitis distinct from that seen in AMA positive $\mathrm{PBC}$ and other autoimmune liver diseases, which may have a different pathogenesis. Further characterisation of the cause and pathogenesis of such patients will be necessary before this classification can be refined.

We thank Dr S P M Fussey for performing some of the immunoassays and J Mariano for conducting the immunofluorescimmunoassays and Mariano for conducting the immunofluorescMinuk, and R J Bailey for allowing us to study their patients and $\mathrm{K}$ Cauch and $\mathrm{V}$ Walker for their expert help in preparing this manuscript.

This work was presented at the AGA in San Fransisco, USA May 1992 and IASL in Brighton, UK June 1992. It was published in abstract form in Hepatology 1992; 16: 568. We are grateful to the Wellcome Trust for supporting this work with a project grant (JMP, SJY, MFB).

1 Walker JG, Doniach D, Roitt IM, Sherlock S. Serological tests in diagnosis of primary biliary cirrhosis. Lancet 1965; i: 827 in

2 Goudie RB, MacSween RNM, Goldberg DM. Serological and histological diagnosis of primary biliary cirrhosis. $\mathcal{F}$ Clin Pathol 1966; 19: 527-38.

3 Klatskin G, Kantor FS. Mitochondrial antibody in primary biliary cirrhosis and other diseases. Ann Intern Med 1972; 77 : 533-41.

4 Munoz LE, Thomas HC, Scheuer PJ, Doniach D, Sherlock S. Is mitochondrial antibody diagnostic of primary biliary cirrhosis? Gut 1981; 22: 136-40.

5 Yeaman SJ, Fussey SPM, Danner DJ, James OFW, Mutimer DJ, Bassendine MF. Primary biliary cirrhosis - identification of two major mitochondrial autoantigens. Lancet 1988; i: $1067-70$.

6 Bassendine MF, Yeaman SJ. Primary biliary cirrhosis: nature of autoantigens. Springer Semin Immunopathol 1990; 12: 73

7 Van de Water J, Fregeau D, Davis P, Ansari A, Danner D, 

Leung $\mathrm{P}$, et al. Autoantibodies of primary biliary cirrhosis recognize dihydrolipoamide acetyltransferase

8 Heseltine L, Turner IB, Fussey SPM, Kelly PJ, James OFW, Yeaman SJ, et al. Primary biliary cirrhosis: quantitation of autoantibodies to purified mitochondrial enzymes and correlation with disease progression. Gastroenterology 1990; 99: 1786-92.

9 Van de Water J, Cooper A, Surh CD, Coppel R, Danner D, Ansari A, et al. Detection of autoantibodies to recombinant mitochondrial proteins in patients with primary biliary cirrhosis. N Engl F Med 1989; 320: 1377-80.

10 Leung PS, Iwayama T, Prindiville T, Chuang DT, Ansari AA Wynn RM, et al. Use of designer recombinant mitochondrial antigens in the diagnosis of primary biliary cirrhosis. Hepatology 1992; 15: 367-72.

11 Beutner EH, Sepulveda MR, Barnett EV. Quantitative studies of immunofluorescent staining. Bull World Health Organ 1968; 39: 587

12 Stanley CJ, Perham RN. Purification of 2-oxo acid dehydrogenase multienzyme complexes from ox heart by a new method. Biochem F 1980; 191: 147-54.

13 Fussey SPM, Guest JR, James OFW, Bassendine MF, Yeaman SJ. Identification and analysis of the major $M 2$ antigens in primary biliary cirrhosis. Proc Natl Acad Sci $U S A$ 1988; 85: 8654-8.

14 Laemmli UK, Favre M. Maturation of the head of bacteriophage T4: DNA packaging events. F Mol Biol 1973; 80: 57599.

15 Fussey SPM, Lindsay JG, Fuller C, Perham RN, Dale S, James OFW, et al. Autoantibodies in primary biliary cirrhosis: analysis of reactivity against eukaryotic and prokaryotic 2-oxo acid dehydrogenase complexes. prokaryotic 2-0xo acid
Hepatology 1991; 13: 467-74.

16 Ludwig, J, Dickson ER, McDonald GSA. Staging of chronic nonsuppurative destructive cholangitis (syndrome of primary biliary cirrhosis). Virchows Arch [A] 1978; 379: 103-12.

17 Walker JG. Immunological tests in liver disease. Ann Clin Biochem 1970; 7: 93-6.

18 Ward AM, Ellis G, Goldberg DM. Immunoglobulin concentrations and autoantibody titres in diseases of the liver and biliary tree. Am f Clin Pathol 1978; 70: 352-8.

19 Bernstein RM, Neuberger JM, Bunn CC, Calendar ME, Hughes GR, Williams R. Diversity of autoantibodies in primary biliary cirrhosis and chronic active hepatitis. Clin
Exp Immunol 1984; 55: 553-60.

20 Powell F, Schroeter AL, Dickson ER. Antinuclear antibodies in primary biliary cirrhosis. Lancet 1984; i: 288-9.

21 Lassoued K, Brenard R, Degos F, Courvalin JC, Andre C, Danon F, et al. Antinuclear antibodies directed to a 200 kilodalton polypeptide of the nuclear envelope in primary biliary cirrhosis. A clinical and immunological study of a series of 150 patients with primary biliary cirrhosis. Gastroenterology 1990; 99: 181-6.

22 Lozano F, Pares A, Borche L, Plana M, Gallart T, Rodes J, et al. Antibodies against nuclear envelope-associated protein in primary biliary cirrhosis. Hepatology 1988; 8: 930-8.

23 Szostecki C, Guldner HH, Netter HJ, Wills H. Isolation and characterisation of cDNA encoding a human nuclear antigen predominantly recognised by autoantibodies from patients with primary biliary cirrhosis. F Immunol 1990; 145: 4338with

24 Kloppel G, Seifert G, Lindner H, Dammermann R, Sack HJ Berg PA. Histopathological features in mixed types of Berg PA. Histopathological features in mixed types of chronic aggressive hepatitis and primat
Virchows Arch [A] 1977; 373: $143-60$.

25 Okuno T, Seto Y, Okanoue T, Takino T. Chronic active hepatitis with histological features of primary biliary cirrhosis. Dig Dis Sci 1987; 32: 775-9.

26 Carrougher JG, Usa M, Shaffer RT. A 33-year old woman with an autoimmune syndrome. Semin $L$ iver Dis 1991; ii: 256-62

27 Ludwig J, Wiesner RH, LaRusso NF. Idiopathic adulthood ductopenia: a cause of chronic cholestatic liver disease and biliary cirrhosis. F Hepatol 1988; 7: 193-9.

28 Galambos JT, Brooks WS. Atypical biliary cirrhosis - or sclerosing cholangitis. 7 Clin Gastroenterol 1980; 2: 43-52.

29 Faa G, Van Eyken P, Demelia L, Vallebona E, Costa V, Desmet VJ. Idiopathic adulthood ductopenia presenting with chronic recurrent cholestasis. $\mathcal{f}$ Hepatol 1991; 12:1420 .

30 Zafrani ES, Metreau J-M, Douvin C, Larrey D, Massari R, Reynes M, et al. Idiopathic biliary ductopenia in adults: a report of five cases. Gastroenterology 1990; 99: 1823-8.

31 Brunner G, Klinge $O$. Ein der chronish-destruierenden nichteitrigen Cholangitis ahnliches Krankheitsbild mit Antikorpern (immuncholangitis). Dtsch Med Wochenschr 1987; 112: 1454-8.

32 Van de Water J, Ansari AA, Surh CD, Coppel R, et al. Evidence for the targeting by 2-oxo-dehydrogenase enzymes in the T cell response of primary biliary cirrhosis. F I mmunol 1991; 146: 89-94. 